OPEN ACCESS

Edited by:

Victor Tapias,

Weill Cornell Medical College, Cornell University, United States

Reviewed by:

Kim Tieu,

Florida International University,

United States

Diego Ruano,

Universidad de Sevilla, Spain Jason Cannon,

Purdue University, United States

Umesh K. Jinwal,

University of South Florida,

United States

${ }^{*}$ Correspondence:

Juan Segura-Aguilar

jsegura@med.uchile.c

Specialty section:

This article was submitted to

Neurodegeneration

a section of the journal

Frontiers in Neuroscience

Received: 26 October 2017 Accepted: 12 February 2018

Published: 13 March 2018

Citation:

Segura-Aguilar $J$ and Huenchuguala $S$

(2018) Aminochrome Induces

Irreversible Mitochondrial Dysfunction

by Inducing Autophagy Dysfunction in

Parkinson's Disease.

Front. Neurosci. 12:106.

doi: 10.3389/fnins.2018.00106

\section{Aminochrome Induces Irreversible Mitochondrial Dysfunction by Inducing Autophagy Dysfunction in Parkinson's Disease}

\author{
Juan Segura-Aguilar $^{1 *}$ and Sandro Huenchuguala ${ }^{2}$ \\ ${ }^{1}$ Molecular and Clinical Pharmacology, Faculty of Medicine, Instituto de Ciencias Biomédicas (ICBM), University of Chile, \\ Santiago, Chile, ${ }^{2}$ Departamento de Ciencias Biológicas y Químicas, Facultad de Ciencia, Universidad San Sebastián, Puerto \\ Montt, Chile
}

Keywords: mitochondrial dysfunction, autophagy dysfunction, lysosomal dysfunction, aminochrome, dopamine, neuromelanin, parkinson's disease, neurodegeneration

\section{MITOCHONDRIAL DYSFUNCTION IN PARKINSON'S DISEASE}

In Parkinson's disease, mitochondrial complex I activity is diminished, and mitochondrial deoxyribonucleic acid (DNA) mutations accumulate (Zhang, 2013). The first evidence that mitochondrial dysfunction was involved in the pathogenesis of Parkinson's disease came from parkinsonism induced by the accidental exposure of drug users to 1-methyl-4-phenyl-1,2,3,4tetrahydropyridine (MPTP), an inhibitor of the mitochondrial complex I of the electron transport chain (Langton et al., 1983; Esteves et al., 2011). Parker et al. (1989) found a significant reduction in the activity of complex I in platelet mitochondria purified from patients with idiopathic Parkinson's disease (Esteves et al., 2011). Further evidence for mitochondrial dysfunction in Parkinson's disease arose from studies developed in the substantia nigra of postmortem brains of patients with the disease, which showed deficiency of complex-I activity (Shapira et al., 1990; Esteves et al., 2011). Genes associated with familial form of the disease has been reported such as pink1, parkin, DJ-1, and CHCHD2 (Kazlauskaite and Muqit, 2015; Meng et al., 2017).

\section{AUTOPHAGY}

Macroautophagy is the most widely studied type of autophagy, where vacuoles with double membranes form, surround cellular elements (such as proteins, lipids, and organelles), and fuse with lysosomes, the enzymes of which degrade the autophagic cargo. Autophagy is controlled by proteins that are encoded by autophagy-related genes (ATGs), ATG1-ATG35. These proteins are organized into complexes that mediate the following steps in the autophagic process: initiation, elongation, maturation, and fusion and degradation (Tan et al., 2014). The pathways that control autophagy are primarily mTOR-dependent and mTOR-independent (mTOR: the mechanistic target of rapamycin, a serine/threonine kinase). mTOR is primarily an inhibitory signal, which participates upstream of the ATG proteins. In the mTOR-independent pathway the autophagy can be directly activated by AMPK [adenosine monophosphate (AMP)-activated protein kinase], leading to direct phosphorylation of ULK1 (serine-threonine-protein kinase that is encoded by the ULK1 gene) and beclin-1 (Tan et al., 2014). 


\section{MITOPHAGY}

The degradation of mitochondria damaged by the autophagic pathway is known as mitophagy and constitutes one of the main mechanisms of cellular homeostasis (Zhang, 2013; Brady and Brady, 2016). Mitochondrial damage causes a decrease in mitochondrial membrane potential or an increase in mitochondrial fission, and both situations activate mitophagy (Brady and Brady, 2016). There are multiple mechanisms by which mitochondria are targeted for degradation in autophagosomes, but the best understood are the pathways of mitophagy induced by PINK/Parkin and BNIP3 (BCL2/adenovirus E1B $19 \mathrm{kDa}$ protein-interacting protein 3), and NIX-dependent mitophagy (Nix: also known as BNIP3L, a BH3-only protein of the BCL-2 pro-apoptotic family). The mitochondrial protein PINK1 [phosphatase and tensin homolog (PTEN)-induced putative kinase 1], a serine-threonine kinase, is unstable due to presenilin-associated rhomboid-like protease activities (PARL). The decrease in mitochondrial membrane potential inhibits PINK1 degradation by PARL. In response to mitochondrial depolarization, PINK stabilizes, and accumulates in the outer mitochondrial membrane (OMM), where it phosphorylates ubiquitin in mitochondrial proteins to recruit autophagic cargo adapters, such as OPTN (Optineurin) and NDP52 (Nuclear dot protein $52 \mathrm{kDa}$ ), which directly bind to light chain 3 (LC3) in the autophagosome leading to degradation of mitochondria within autophagolysosomes (Springer and Macleod, 2016). PINK1 also recruits the E3 ubiquitin ligase Parkin and ubiquitin-specific substrates in the OMM, including VDAC (voltage-dependent anion-selective channel), Miro, and Mitofusin-2 to amplify the signal initiated by PINK (Springer and Macleod, 2016). Mutations in PARK2 (Parkin) and PARK6 (PINK1) have been independently linked to familial cases of Parkinson's disease, associating defects in mitophagy with the degeneration of dopaminergic neurons, a major feature of Parkinson's disease (Pikrell and Youle, 2015; Springer and Macleod, 2016).

The clearance of mitochondria damaged by mitophagy prevents the accumulation of dysfunctional mitochondria and can also induce mitochondrial biogenesis, increasing cell survival. On the contrary, the decrease in mitophagy occurring during aging, for example, prevents both the removal of damaged mitochondria and alters mitochondrial biogenesis, which causes the progressive accumulation of dysfunctional mitochondria (Zhang, 2013; Palikaras et al., 2015).

The dysfunction of the autophagic/lysosomal pathway is associated with mitochondrial dysfunction, which may be due to the decrease in the autophagic degradation of the dysfunctional mitochondria. For example, Wu et al. (2009) reported that deletion of an ATG7 autophagic protein in mice causes mitochondrial dysfunction, characterized by decreased mitochondrial oxygen uptake rate and increased levels of reactive oxygen species in muscle fibers and pancreatic beta cells. On the other hand, lysosomal storage disorders are associated with mitochondrial dysfunctions, which include changes in mitochondrial morphology, decreased mitochondrial membrane potential, decreased ATP production, and increased generation of reactive oxygen species (De la Mata et al., 2016; Plotegher and Dushen, 2017). The loss of lysosomal glucocerebrosidase enzyme activity causes lysosomal dysfunction (Bae et al., 2015). In turn, glucocerebrosidase deficiency is associated with mitochondrial dysfunction (Gregg and Schapira, 2016). Mitochondrial dysfunction may also induce lysosomal dysfunction, resulting in a vicious circle (Plotegher and Dushen, 2017). In the context of Parkinson's disease, mitochondrial dysfunction and lysosomal dysfunction may be connected by the oxidation of dopamine. In fact, it has recently been reported that dopamine oxidation mediates mitochondrial and lysosomal dysfunction in both sporadic and familial Parkinson's disease (Burbulla et al., 2017).

\section{DOPAMINE OXIDATION TO NEUROMELANIN}

Dopamine oxidation to neuromelanin is a sequential pathway where several ortho(o)-quinones are formed: dopamine $\rightarrow$ dopamine $\quad o$-quinone $\rightarrow$ aminochrome $\rightarrow 5,6$ indolequinone $\rightarrow$ neuromelanin. Dopamine $o$-quinone is converted to aminochrome with a constant rate of $0.15^{\mathrm{s}-1}$ at physiological $\mathrm{pH}$ (Tse et al., 1976). Oxidation of dopamine with tyrosinase to dopamine $o$-quinone in the presence of mitochondria induce the formation adduct with a long list of proteins but in the same study incubation of SH-SY5Y cells with dopamine only few adducts were detected, questioning the feasibility of dopamine $o$-quinone to be responsible for dopamine neurotoxicity (Van Laar et al., 2009). Dopaminochrome, which structure has not be determined, induces adducts with alpha-synuclein (Norris et al., 2005) and 5,6-indolequinone induced adducts with alpha synuclein (Bisaglia et al., 2007). Aminochrome is the most stable $o$-quinone.

\section{AMINOCHROME AND MITOCHONDRIAL DYSFUNCTION}

For a long time, it has been accepted that mitochondrial dysfunction is involved in the degeneration of the nigrostriatal neurons containing neuromelanin in Parkinson's disease, but the question of what induces mitochondrial dysfunction inside of dopaminergic neurons containing neuromelanin remains. A possible candidate is aminochrome, an $o$-quinone formed during dopamine oxidation to neuromelanin (Segura-Aguilar et al., 2014, 2016; Herrera et al., 2017; Segura-Aguilar, 2017a,b). Aminochrome is an endogenous neurotoxin that induces mitochondrial dysfunction by inhibiting complex I and decreasing ATP levels in different cell lines (Arriagada et al., 2004; Aguirre et al., 2012; Huenchuguala et al., 2017). Aminochrome induces mitochondrial dysfunction in rat brain, resulting in a significant decrease in ATP levels, which explains a significant the decrease in dopamine release and amount of synaptic vesicles at the synaptic cleft. Both the axonal transport of neurotransmitter vesicles to the terminals and dopamine release require ATP (Herrera et al., 2016). Interestingly, aminochrome also induces other mechanisms related to the degeneration of dopaminergic 
neurons containing neuromelanin, such as protein degradation dysfunction of both lysosomal and proteasomal systems (Zafar et al., 2006; Huenchuguala et al., 2014); aggregation of alphasynuclein to neurotoxic oligomers (Muñoz et al., 2015); neuroinflammation (Santos et al., 2017); oxidative (Arriagada et al., 2004); and endoplasmic reticulum stress (Xiong et al., 2014). The oxidation of dopamine to neuromelanin is a normal pathway, because healthy seniors have intact dopaminergic neurons containing neuromelanin (Zecca et al., 2002; Zucca et al., 2017). A relationship between neuromelanin content and loss of dopaminergic neurons containing neuromelanin has been reported. The level of neuromelanin in substantia nigra pars compacta was 10 times higher than in ventral tegmental area of control subjects. The loss of dopaminergic neurons containing neuromelanin in Parkinson's disease patients was found to be $47 \%$ in comparison $0 \%$ in ventral tegmental area (Schwarz et al., 2017). The 10-fold lower amount of neuromelanin in ventral tegmental area results in lower amount of aminochrome, explaining why these neurons were intact in this study (Schwarz et al., 2017). Neuromelanin formation depends on VMAT-2 expression because dopamine is complete stable inside of monoaminergic vesicles where the $\mathrm{pH}$ is around 5.3 due to dopamine uptake is coupled to an ATPase proton pump (Sulzer et al., 2000; Herrera et al., 2017; Segura-Aguilar, 2017a,b). An inverse relationship between VMAT-2 expression level and neuromelanin content in human midbrain dopamine neurons has been reported (Liang et al., 2014). The reason why aminochrome is not neurotoxic in dopaminergic neurons containing neuromelanin in healthy seniors is because the enzymes DT-diaphorase and glutathione transferase M2-2 (GSTM2) prevent aminochrome neurotoxicity (Lozano et al., 2010; Huenchuguala et al., 2014, 2016; Segura-Aguilar, 2015, 2017a; Herrera-Soto et al., 2017; Muñoz and Segura-Aguilar, 2017) (Figure 1). DT-diaphorase is expressed both in dopaminergic neurons and astrocytes, but GSTM2 is only expressed in astrocytes. However, GSTM2 protects both astrocytes and dopaminergic neurons against aminochrome neurotoxicity because astrocytes secrete GSTM2 and dopaminergic neurons internalize GSTM2 into the cytosol (Cuevas et al., 2015; Segura-Aguilar et al., 2016; SeguraAguilar, 2017b,c). The question is why GSTM2 and DTdiaphorase are not protecting dopaminergic neurons containing

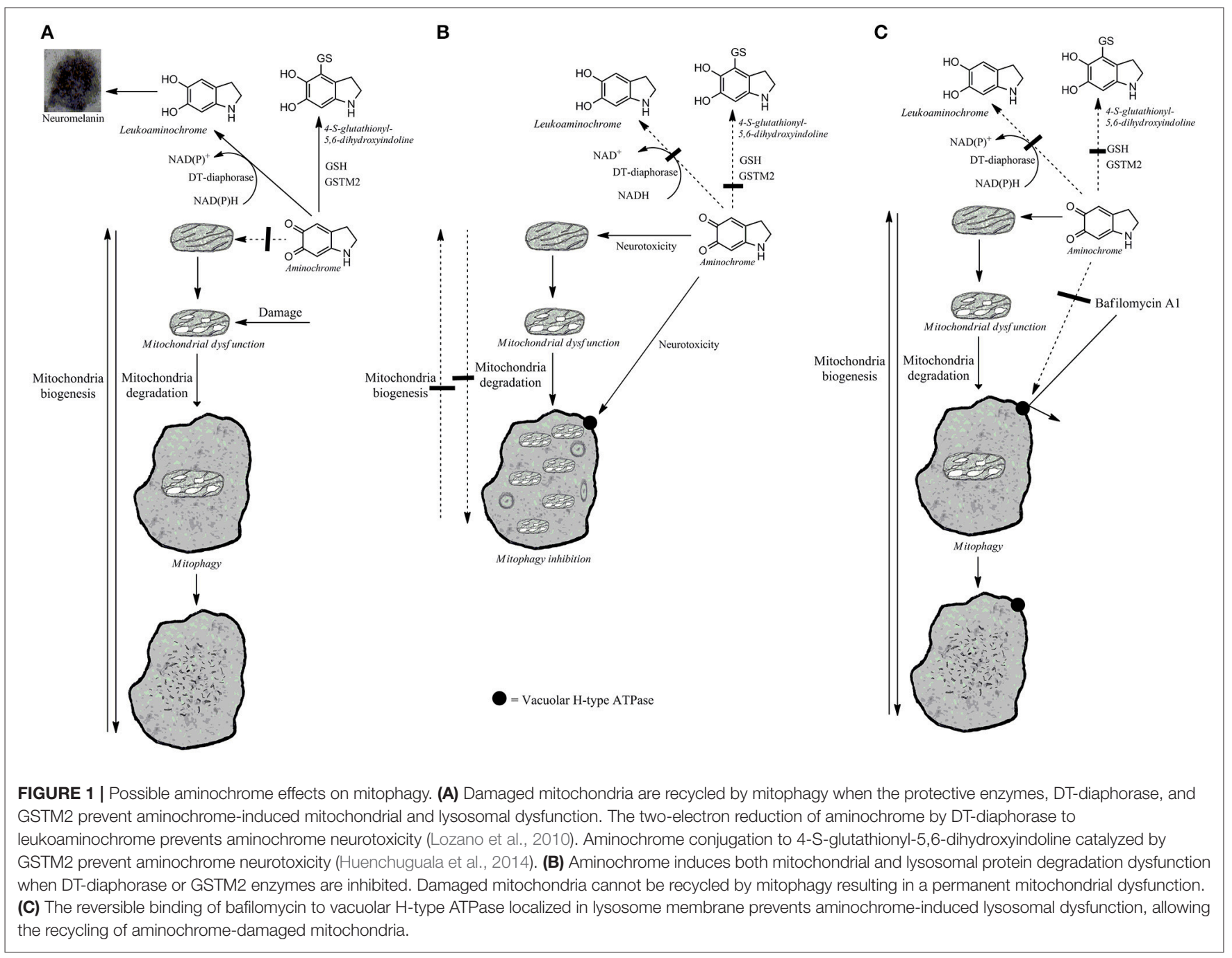


neuromelanin in Parkinson's disease patients. A possible explanation is that an over production of aminochrome surpass the enzyme capacity $(\mathrm{Km})$ to prevent its neurotoxic effects or for some unknown reason these enzyme are down regulated or inhibited.

\section{THE IMPORTANCE OF MITOPHAGY IN PREVENTING MITOCHONDRIAL DYSFUNCTION}

In general, mitochondrial dysfunction activates mitophagy as a defense mechanism to remove damaged mitochondria (Brady and Brady, 2016). Mitophagy seems to play a key homeostatic role in mitochondrial quality control (Tan and Wong, 2017). It has been proposed that age-dependent deterioration of mitophagy both inhibits the removal of damaged mitochondria and impairs mitochondrial biogenesis (Palikaras et al., 2017). The problem is when mitochondrial dysfunction is induced by a neurotoxin that induces both mitochondrial and autophagy dysfunction. Aminochrome-induced mitochondrial dysfunction is irreversible because aminochrome also induces autophagy dysfunction by preventing the fusion between autophagy vacuoles and lysosome dysfunction by increasing their $\mathrm{pH}$ (Huenchuguala et al., 2014). Recently, we have demonstrated that mitophagy plays a key role in reversing aminochromeinduced mitochondrial dysfunction (Huenchuguala et al., 2017). The pre-incubation of cells with bafilomycin A1, a reversible inhibitor of lysosomal vacuolar-type $\mathrm{H}^{+}$-ATPase, before the incubation with aminochrome restores ATP levels, mitochondrial membrane potential, and mitophagy, and decreases cell death (Huenchuguala et al., 2017). Aminochrome cannot form adduct with vacuolar-type $\mathrm{H}^{+}$-ATPase because bafilomycin A1 pre-incubated with the cells prevents it. Then after a while, bafilomycin dissociates from ATPase but

\section{REFERENCES}

Aguirre, P., Urrutia, P., Tapia, V., Villa, M., Paris, I., Segura-Aguilar, et al. (2012). The dopamine metabolite aminochrome inhibits mitochondrial complex I and modifies the expression of iron transporters DMT1 and FPN1. Biometals 25, 795-803. doi: 10.1007/s10534-012-9525-y

Arriagada, C., Paris, I., Sanchez de las Matas, M. J., Martinez-Alvarado, P., Cardenas, S., Castañeda, P., et al. (2004). On the neurotoxicity mechanism of leukoaminochrome $o$-semiquinone radical derived from dopamine oxidation: mitochondria damage, necrosis, and hydroxyl radical formation. Neurobiol. Dis. 16, 468-477. doi: 10.1016/j.nbd.2004.03.014

Bae, E.-J., Yang, N. Y., Lee, C., Lee, H.-Y., Kim, S., and Sardi, S. P. (2015). Loss of glucocerebrosidase 1 activity causes lysosomal dysfunction and $\alpha$-synuclein aggregation. Exp. Mol. Med. 47:e153. doi: 10.1038/emm.2014.128

Bisaglia, M., Mammi, S., and Bubacco, L. (2007). Kinetic and structural analysis of the early oxidation products of dopamine: analysis of the interactions with alpha-synuclein. J. Biol. Chem. 282, 15597-15605. doi: 10.1074/jbc.M610893200

Brady, A. H., and Brady, N. R. (2016). Mitophagy programs: mechanisms and physiological implications of mitochondrial targeting by autophagy. Cell. Mol. Life Sci. 73, 775-795. doi: 10.1007/s00018-015-2087-8

Burbulla, L. F., Song, P., Mazzulli, J. R., Zampese, E., Wong, Y. C., Jeon, S., et al. (2017). Dopamine oxidation mediates mitochondrial and lysosomal dysfunction in Parkinson's disease. Science 357, 1255-1261. doi: $10.1126 /$ science.aam9080 aminochrome is not as stable to form adducts with ATPase after bafilomycin has dissociated. When aminochrome is prevented from binding to lysosomal vacuolar-type $\mathrm{H}^{+}$-ATPase it can bind other proteins or can be reduced by flavoenzymes. These experiments support (i) the enormous importance of mitophagy in preventing mitochondrial dysfunction and (ii) the irreversible neurotoxic action of aminochrome, as a consequence of its ability to be neurotoxic by inducing both mitochondria and mitophagy dysfunction (Figure 1).

\section{CONCLUSION}

Mitochondrial dysfunction seems to play an important role in the loss of dopaminergic neurons containing neuromelanin in the nigrostriatal system in Parkinson's disease. The accurate removal of dysfunctional mitochondria by mitophagy is essential for keeping control over normal mitochondrial function in neurons. The endogenous neurotoxin aminochrome induces an irreversible mitochondrial dysfunction because it induces both mitochondrial and lysosomal protein degradation dysfunction. However, when bafilomycin A1 prevented aminochrome-dependent lysosomal dysfunction, the normal mitochondrial function was recovered, highlighting the essential role of the lysosomal protein degradation system in the prevention of mitochondrial dysfunction in Parkinson's disease.

\section{AUTHOR CONTRIBUTIONS}

JS-A: design and write the paper; $\mathrm{SH}$ : write a part of the paper.

\section{FUNDING}

This work was funded by FONDECYT 1170033.

Cuevas, C., Huenchuguala, S., Muñoz, P., Villa, M., Paris, I., Mannervik, B., et al. (2015). Glutathione transferase-M2-2 secreted from glioblastoma cell protects SH-SY5Y cells from aminochrome neurotoxicity. Neurotox. Res. 27, 217-228. doi: 10.1007/s12640-014-9500-1

De la Mata, M., Cotán, D., Villanueva-Paz, M., de Lavera, I., Alvarez-Córdoba, M., Luzón-Hidalgo, R., et al. (2016). Mitochondrial dysfunction in lysososomal storage disorders. Diseases 4:31. doi: 10.3390/diseases4040031

Esteves, A. R., Arduíno, D. M., Silva, D. F. F., Oliveira, C. R., and Cardoso, S. M. (2011). Mitochondrial dysfunction: the road to alpha-synuclein oligomerization in PD. Parkinson's Dis. 2011:693761. doi: 10.4061/2011/693761

Gregg, M. E., and Schapira, H. (2016). Mitochondrial dysfunction associated with glucocerebrosidase deficiency. Neurobiol. Dis. 90, 43-50. doi: 10.1016/j.nbd.2015.09.006

Herrera, A., Muñoz, P., Paris, I., Díaz-Veliz, G., Mora, S., Inzunza, J., et al. (2016). Aminochrome induces dopaminergic neuronal dysfunction: a new animal model for Parkinson's disease. Cell. Mol. Life Sci. 73, 3583-3597. doi: $10.1007 / \mathrm{s} 00018-016-2182-5$

Herrera, A., Muñoz, P., Steinbusch, H. W. M., and Segura-Aguilar, J. (2017). Are dopamine oxidation metabolites involved in the loss of dopaminergic neurons in the nigrostriatal system in Parkinson's Disease? ACS Chem. Neurosci. 8, 702-711. doi: 10.1021/acschemneuro.7b00034

Herrera-Soto, A., Díaz-Veliz, G., Mora, S., Muñoz, P., Henny, P., Steinbusch, H. W. M., et al. (2017). On the role of DT-Diaphorase inhibition in aminochrome-induced neurotoxicity In Vivo. Neurotox. Res. 32, 134-140. doi: 10.1007/s12640-017-9719-8 
Huenchuguala, S., Muñoz, P., and Segura-Aguilar, J. (2017). The importance of mitophagy in maintaining mitochondrial function in U373MG Cells. Bafilomycin A1 restores aminochrome-induced mitochondrial damage. ACS Chem. Neurosci. 8, 2247-2253. doi: 10.1021/acschemneuro.7b00152

Huenchuguala, S., Muñoz, P., Graumann, R., Paris, I., and Segura-Aguilar, J. (2016). DT-diaphorase protects astrocytes from aminochrome-induced toxicity. Neurotoxicology 55, 10-12. doi: 10.1016/j.neuro.2016.04.014

Huenchuguala, S., Muñoz, P., Zavala, P., Villa, M., Cuevas, C., Ahumada, U., et al. (2014). Glutathione transferase mu 2 protects glioblastoma cells against aminochrome toxicity by preventing autophagy and lysosome dysfunction. Autophagy 10, 618-630. doi: 10.4161/auto.27720

Kazlauskaite, A., and Muqit, M. M. (2015). PINK1 and Parkin - mitochondrial interplay between phosphorylation and ubiquitylation in Parkinson's disease. FEBS J. 282, 215-223. doi: 10.1111/febs.13127

Langton, J. M., Ballard, P., Tetrud, J. W., and Irwin, I. (1983). Chronic parkinsonism in humans due to a product of meperidine-analog synthetisis. Science 219, 979-980. doi: 10.1126/science.6823561

Liang, C. L., Nelson, O., Yazdani, U., Pasbakhsh, P., and German, D. C. (2014). Inverse relationship between the contents of neuromelanin pigment and the vesicular monoamine transporter-2: human midbrain dopamine neurons. J. Comp. Neurol. 473, 97-106. doi: 10.1002/cne.20098

Lozano, J., Muñoz, P., Nore, B. F., Ledoux, S., and Segura-Aguilar, J. (2010). Stable expression of short interfering RNA for DT-diaphorase induces neurotoxicity. Chem. Res. Toxicol. 23, 1492-1496. doi: 10.1021/tx100182a

Meng, H., Yamashita, C., Shiba-Fukushima, K., Inoshita, T., Funayama, M., Sato, S., et al. (2017). Loss of Parkinson's disease-associated protein CHCHD2 affects mitochondrial crista structure and destabilizes cytochrome c. Nat. Commun. 8:15500. doi: $10.1038 /$ ncomms 15500

Muñoz, P. S., and Segura-Aguilar, J. (2017). DT-diaphorase protects against autophagy induced by aminochrome-dependent alpha-synuclein oligomers. Neurotox. Res. 32, 362-367. doi: 10.1007/s12640-017-9747-4

Muñoz, P., Cardenas, S., Huenchuguala, S., Briceño, A., Couve, E., Paris, I., et al. (2015). DT-Diaphorase prevents aminochrome-induced alphasynuclein oligomer formation and neurotoxicity. Toxicol. Sci. 145, 37-47. doi: $10.1093 /$ toxsci/kfv016

Norris, E. H., Giasson, B. I., Hodara, R., Xu, S., Trojanowski, J. Q., Ischiropoulos, H., et al. (2005). Reversible inhibition of alpha-synuclein fibrillization by dopaminochrome-mediated conformational alterations. J. Biol. Chem. 280, 21212-22129. doi: 10.1074/jbc.M412621200

Palikaras, K., Daskalaki, I., Markaki, M., and Tavernarakis, N. (2017). Mitophagy and age-related pathologies: development of new therapeutics by targeting mitochondrial turnover. Pharmacol. Ther. 178, 157-174. doi: 10.1016/j.pharmthera.2017.04.005

Palikaras, K., Lionaki, E., and Tavernarakis, N. (2015). Coordination of mitophagy and mitochondrial biogenesis during ageing in C. elegans. Nature 521, 525-528. doi: 10.1038/nature14300

Parker, W. D. Jr., Boyson, S. J., and Parks, K. (1989). Abnormalities of the electron transport chain in idiopathic Parkinson's disease. Ann. Neurol. 26, 719-723.

Pikrell, A. M., and Youle, R. J. (2015). The roles of PINK1, parkin, and mitochondrial fidelity in Parkinson's disease. Neuron 85, 257-273. doi: 10.1016/j.neuron.2014.12.007

Plotegher, N., and Dushen, M. R. (2017). Mitochondrial dysfunction and neurodegeneration in lysosomal storage disorders. Trends Mol. Med. 23, 116-134. doi: 10.1016/j.molmed.2016.12.003

Santos, C. C., Araújo, F. M., Ferreira, R. S., Silva, V. B., Silva, J. H. C., Grangeiro, M. S., et al. (2017). Aminochrome induces microglia and astrocyte activation. Toxicol. In Vitro 42, 54-60. doi: 10.1016/j.tiv.2017.04.004

Schwarz, S. T., Xing, Y., Tomar, P., Bajaj, N., and Auer, D. P. (2017). In vivo assessment of brainstem depigmentation in Parkinson disease: potential as a severity marker for multicenter studies. Radiology 283, 789-798. doi: $10.1148 /$ radiol.2016160662

Segura-Aguilar, J. (2015). A new mechanism for protection of dopaminergic neurons mediated by astrocytes. Neural Regen. Res. 10, 1225-1227. doi: $10.4103 / 1673-5374.162750$

Segura-Aguilar, J. (2017a). Aminochrome as preclinical model for Parkinson's disease. Oncotarget 8, 45036-45037. doi: 10.18632/oncotarget.18353

Segura-Aguilar, J. (2017b). On the role of endogenous neurotoxins and neuroprotection in Parkinson's disease. Neural Regen. Res 12, 897-901. doi: 10.4103/1673-5374.208560
Segura-Aguilar, J. (2017c). Commentary: gene therapy: a promising approach for neuroprotection in Parkinson's Disease? Front. Neuroanat. 11:40. doi: $10.3389 /$ fnana.2017.00040

Segura-Aguilar, J., Paris, I., Muñoz, P., Ferrari, E., Zecca, L., and Zucca, F. A. (2014). Protective and toxic roles of dopamine in Parkinson's disease. J. Neurochem. 129, 898-915. doi: 10.1111/jnc.12686

Segura-Aguilar, J., Muñoz, P., Paris, I. (2016). Aminochrome as new preclinical model to find new pharmacological treatment that stop the development of Parkinson's disease. Curr. Med. Chem. 23, 346-359. doi: 10.2174/0929867323666151223094103

Shapira, A. H. V., Cooper, J. M., Dexter, D., Clark, J. B., Jenner, P., and Marsden, C. D. (1990). Mitochondrial complex I deficiency in Parkinson's disease. J. Neurochem. 54, 823-827. doi: 10.1111/j.1471-4159.1990. tb02325.x

Springer, M. Z., and Macleod, K. F. (2016). Mitophagy, mechanisms and role in human disease. J. Pathol. 240, 253-255. doi: 10.1002/path.4774

Sulzer, D., Bogulavsky, J., Larsen, K. E., Behr, G., Karatekin, E., Kleinman, M. H., et al. (2000). Neuromelanin biosynthesis is driven by excess cytosolic catecholamines not accumulated by synaptic vesicles. Proc. Natl. Acad. Sci. U.S.A. 97, 11869-11874. doi: 10.1073/pnas.97.22.11869

Tan, C. C., Yu, J.-T., Meng, S. T., Jiang, T., and Zhu, X.-C. (2014). Autophagy in aging and neurodegenerative diseases: implications for pathogenesis and therapy. Neurobiol. Aging 35, 941-957. doi: 10.1016/j.neurobiolaging.2013.11.019

Tan, S., and Wong, E. (2017). Mitophagy transcriptome: mechanistic insights into polyphenol-mediated mitophagy. Oxid. Med. Cell. Longev. 2017:9028435. doi: $10.1155 / 2017 / 9028435$

Tse, D. C. S., McCreery, R. L., and Adams, R. N. (1976). Potential oxidative pathways of brain catecholamines. J. Med. Chem. 19, 37-34. doi: 10.1021/jm00223a008

Van Laar, V. S., Mishizen, A. J., Cascio, M., and Hastings, T. G. (2009). Proteomic identification of dopamine-conjugated proteins from isolated rat brain mitochondria and SH-SY5Y cells. Neurobiol. Dis. 3, 487-500. doi: 10.1016/j.nbd.2009.03.004

Wu, J. J., Quijano, C., Chen, E., Liu, H., Cao, L., Fergusson, M. M., et al. (2009). Mitochondrial dysfunction and oxidative stress mediate the physiological impairment induced by disruption of autophagy. Aging 1, 425-437. doi: 10.18632/aging. 100038

Xiong, R., Siegel, D., and Ross, D. (2014). Quinone-induced protein handling changes: implications for major protein handling systems in quinone-mediated toxicity. Toxicol. Appl. Pharmacol. 280, 285-295. doi: 10.1016/j.taap.2014.08.014

Zafar, K. S., Siegel, D., and Ross, D. (2006). A potential role for cyclized quinones derived from dopamine, DOPA, and 3,4-dihydroxyphenylacetic acid in proteasomal inhibition. Mol. Pharmacol. 70, 1079-1086. doi: $10.1124 / \mathrm{mol} .106 .024703$

Zecca, L., Fariello, R., Riederer, P., Sulzer, D., Gatti, A., and Tampellini, D. (2002). The absolute concentration of nigral neuromelanin, assayed by a new sensitive method, increases throughout the life and is dramatically decreased in Parkinson's disease. FEBS Lett. 510, 216-220. doi: 10.1016/S0014-5793(01)03269-0

Zhang, J. (2013). Autophagy and mitophagy in cellular damage control. Redox Biol. 1, 19-23. doi: 10.1016/j.redox.2012.11.008

Zucca, F. A., Segura-Aguilar, J., Ferrari, E., Muñoz, P., Paris, I., Sulzer, D., et al. (2017). Interactions of iron, dopamine and neuromelanin pathways in brain aging and Parkinson's disease. Prog. Neurobiol. 155, 96-119. doi: 10.1016/j.pneurobio.2015.09.012

Conflict of Interest Statement: The authors declare that the research was conducted in the absence of any commercial or financial relationships that could be construed as a potential conflict of interest.

Copyright (C) 2018 Segura-Aguilar and Huenchuguala. This is an open-access article distributed under the terms of the Creative Commons Attribution License (CC $B Y)$. The use, distribution or reproduction in other forums is permitted, provided the original author(s) and the copyright owner are credited and that the original publication in this journal is cited, in accordance with accepted academic practice. No use, distribution or reproduction is permitted which does not comply with these terms. 